\title{
Renal Handling of Carnitine in Secondary Carnitine Deficiency Disorders
}

\author{
CHARLES A. STANLEY, GERARD T. BERRY, MICHAEL J. BENNETT, STEVEN M. WILLI, \\ WILLIAM R. TREEM, AND DANIEL E. HALE \\ Divisions of Endocrinology/Diabetes [C.A.S., S.M.W., D.E.H.] and Metabolism [G.T.B.J. The Children's \\ Hospital of Philadelphia, Department of Pediatrics, University of Pennsylvania School of Medicine, \\ Philadelphia, Pennsylvania 19104; Metabolic Disease Center, Baylor University Medical Center, \\ Dallas, Texas 75244 [M.J.B.]; and Division of Pediatric Gastroenterology, Hartford Hospital, \\ Department of Pediatrics, University of Connecticut School of Medicine, \\ Farmington, Connecticut 06115 [W.R.T.]
}

\begin{abstract}
Reduced plasma and tissue concentrations of carnitine, a cofactor required for fatty acid oxidation, are present in patients with inherited disorders of mitochondrial acyl-CoA oxidation that are associated with accumulations of acylcarnitines. To determine whether the secondary carnitine deficiency in these patients is due to excessive urinary loss of acylcarnitines, the development of carnitine deficiency was examined in patients with four different acyl-CoA oxidation disorders, including medium-chain and long-chain fatty acyl-CoA dehydrogenase deficiencies, isovaleric acidemia, and propionic acidemia. After a 3-mo period of treatment with oral carnitine to raise plasma total carnitine concentrations to or above normal, patients were started on a carnitine-free diet and the changes in plasma total and free carnitine levels and urinary total and free carnitine excretion were followed for $5 \mathrm{~d}$. Patients with all four disorders showed a return of plasma carnitine levels and urinary carnitine excretion to baseline within 2 to $4 \mathrm{~d}$. The rapidity of these changes could not be explained solely by excessive acylcarnitine wasting. Continued excretion of free carnitine in all patients indicated the additional presence of an impairment in renal transport of free carnitine. Consistent with this interpretation, estimates of renal thresholds for free carnitine gave values that were less than that for a control child in all four disorders and ranged as low as one half those reported in normal individuals. These results suggest that secondary carnitine deficiency in the acyl-CoA oxidation disorders is due to indirect as well as direct effects of accumulated acylcarnitines. Lowered renal free carnitine thresholds in these patients may reflect inhibition of free carnitine transport in the kidney by acylcarnitines. (Pediatr Res 34: 89-97, 1993)
\end{abstract}

\section{Abbreviations}

IVA, isovaleric acidemia

LCAD, long-chain acyl-CoA dehydrogenase deficiency

MCAD, medium-chain acyl-CoA dehydrogenase deficiency MMA, methylmalonic aciduria

PA, propionic acidemia

Received September 14, 1992; accepted February 26, 1993

Correspondence and reprint requests: Charles A. Stanley, M.D., Endocrinology/ Diabetes Division. The Children's Hospital of Philadelphia, 34th Street and Civic Center Blvd., Philadelphia, PA 19104.

Supported in part by grants from the National Institutes of Health (RR-00240, NS-17752) and Sigma Tau Pharmaceuticals.
Carnitine is a derivative of lysine that plays an essential role in long-chain fatty acid oxidation by transporting fatty acids into mitochondria as their acylcarnitine esters (1). Secondary carnitine deficiency has been observed in patients with a large number of genetic diseases that are associated with impaired oxidation of acyl-CoA intermediates in mitochondria (2). These include defects in straight-chain fatty acid oxidation, such as MCAD (3), and branched-chain organic acid oxidation, such as IVA (4). When patients with these disorders are well, they have reduced plasma and tissue total carnitine concentrations that are $25-50 \%$ of normal and an increased ratio of plasma acylcarnitines to total carnitine. There is controversy about the role of carnitine treatment in these disorders, in part because the mechanism of the secondary carnitine deficiency is unclear.

The basis of the alteration in plasma and tissue carnitine concentrations is likely to be related to the fact that the acyl$\mathrm{CoA}$ intermediates that accumulate within the mitochondria in these disorders can form acylcarnitine esters through the action of one or more carnitine acyltransferases. Because some of the defects are associated with the appearance of abnormal acylcarnitines in urine (5), the mechanism most commonly proposed is that the carnitine deficiency is caused by excessive urinary excretion of these acylcarnitine esters. Such a mechanism has been shown to account for the carnitine deficiency induced by medications containing pivalate, a branched-chain fatty acid that is excreted as pivaloylcarnitine $(6,7)$. However, some defects with secondary carnitine deficiency, such as LCAD, are not associated with abnormal acylcarnitines in the urine $(5,8)$. A second possible mechanism is that renal conservation of free carnitine is impaired, leading to carnitine deficiency in a manner similar to that seen in patients with a genetic defect in carnitine transport $(9,10)$ or the renal Fanconi syndrome (11). This mechanism is supported by observations that acylcarnitines inhibit the transport of free carnitine in cultured cells (10) and that the renal threshold for free carnitine is decreased in patients with MCAD $(2,12,13)$. Previous studies of the initial stages in the development of carnitine deficiency in patients given pivalate and in patients with a genetic defect in carnitine transport have provided the clearest method of distinguishing between these two different mechanisms $(6,9)$.

The purpose of the present studies was to test the hypothesis that acylcarnitine wastage, similar to that seen with pivalate administration, is responsible for secondary carnitine deficiency in genetic defects in acyl-CoA oxidation. The evolution of carnitine deficiency after a period of carnitine repletion was examined in patients with four different disorders. These included two fatty acid $\beta$-oxidation enzyme defects, MCAD and LCAD, and two organic acid oxidation disorders, IVA and PA. The responses to acute withdrawal of high-dose carnitine replacement therapy 
indicate that patients with all of these acyl-CoA oxidation disorders have impaired renal conservation of free carnitine as well as increased acylcarnitine excretion. This impairment may make an important contribution to their secondary carnitine deficiency.

\section{PATIENTS AND METHODS}

Studies of plasma carnitine concentrations and urinary carnitine excretion in the untreated state were carried out in patients while they were well and on their usual diets. There were six patients with MCAD, aged 1-11 y; one patient with LCAD, aged $1 \mathrm{y}$; two patients with IVA, aged 1 and $5 \mathrm{y}$; two patients with MMA, aged 3 and $5 \mathrm{y}$; and one patient with PA, aged $2 \mathrm{y}$. As shown in Table 1, two of the MCAD patients, the LCAD patient, and the two IVA patients also participated several years later in the carnitine withdrawal protocol described below. Seven control children, aged 2-11 y, were studied during elective admissions to evaluate possible hypoglycemia. Plasma was obtained 4-12 h postprandial and urine was collected for 12-24 h.

Studies of responses to withdrawal of oral carnitine treatment were carried out in two patients with MCAD (case 1, 6 y old; case 2, 16 y old); two patients with LCAD (case 1, 2 y old; case 2, 7 y old); three patients with IVA (case 1, 3 y old; case 2, 6 y old; case 3, 10 y old); and one patient with PA (aged $3 \mathrm{y}$ ). The two MCAD patients were homozygous for the common $985_{A \rightarrow G}$ mutation (14-16). The response to carnitine withdrawal was also studied in one control child, aged $5 \mathrm{y}$, who had been on supplemental carnitine for over $1 \mathrm{y}$. Before treatment, plasma free (35 $\mu \mathrm{mol} / \mathrm{L})$ and total $(42 \mu \mathrm{mol} / \mathrm{L})$ carnitine concentrations had been normal. For comparison, results from a previously reported 6-mo-old infant with a genetic defect in carnitine transport (9) were included. The effect of a carnitine-free diet for $5 \mathrm{~d}$ was studied in 10 healthy adult volunteers, six males and four females. The carnitine-free diet excluded all animal products: meat, milk, eggs, and cheese. All studies were approved by the Institutional Review Board of The Children's Hospital of Philadelphia.

Before studies of the response to carnitine withdrawal, patients were treated with oral L-carnitine, $1-2 \mathrm{~g} / \mathrm{d}(50-100 \mathrm{mg} / \mathrm{kg} / \mathrm{d})$, in two or three divided doses for 3 or more mo. They were admitted to the Clinical Research Center and continued on therapy for the first $24 \mathrm{~h}$. The last dose of oral L-carnitine was given at 0800 $\mathrm{h}$, and a carnitine-free diet was begun and continued for 4-6 d. Blood was collected at 0800 and $2000 \mathrm{~h}$ daily (or more frequently) for plasma carnitine determination; urine was collected in 12-h aliquots for urinary carnitine determination. Based on the rates of total carnitine excretion, absorption of oral carnitine dose was calculated to be $5-10 \%$ in most patients; it was $30 \%$ in IVA case $3,20 \%$ in the disease control infant with a defect in carnitine transport (9), and $18 \%$ in the control normal child.

Free carnitine concentrations were assayed in plasma and urine by a radioenzymatic method (17). Total carnitine was measured by this assay after alkaline hydrolysis and acylcarnitine calculated as the difference between free and total carnitine. The normal range for plasma total carnitine in our lab is $40-60 \mu \mathrm{mol} / \mathrm{L}$ in children and adults. The rates of urinary carnitine excretion were expressed as $\mu \mathrm{mol} / \mathrm{g}$ creatinine to minimize problems with incomplete collections of urine. When expressed in this manner, rates of total carnitine excretion in control children were similar over the age range of $1-11$ y but were higher than in control adults (see Results). The renal clearance of free carnitine was expressed as the percentage of the filtered load (urine free carnitine/plasma free carnitine $\times$ plasma creatinine/urine creatinine $\times 100$ ). Free carnitine clearance was $2.4 \pm 1.4 \%$ in the control children and $1.8 \pm 0.8 \%$ in the control adults (mean \pm SD). The renal threshold for free carnitine was estimated from the results of the withdrawal studies as the plasma free carnitine level at which the clearance of free carnitine was no longer clearly abnormal (i.e. within 2 SD of the mean of control children or less than $5 \%$ of the filtered load). Clearance rates were plotted against plasma concentrations and the intercept with a clearance rate of $5 \%$ estimated by linear interpolation. The normal renal threshold for free carnitine, based on a more precise method using short-term carnitine infusions, is similar to normal plasma concentrations of free carnitine (12).

\section{RESULTS}

Table 1 shows the concentrations of plasma carnitine and rates of urinary carnitine excretion in 12 patients with five genetic defects in acyl-CoA oxidation obtained while they were not receiving carnitine treatment and compares them with values found in control children. The reduction in plasma total carnitine concentration was greater in the MCAD, LCAD, and IVA patients than in the MMA and PA patients. All of the disorders were associated with an increase in the proportion of plasma carnitine that was esterified; one patient each with MMA and PA had abnormally high absolute concentrations of plasma acylcarnitine. In none of the disorders did the rate of urinary total carnitine excretion exceed the mean plus 2 SD found in control children $(460 \mu \mathrm{mol} / \mathrm{L})$. Mean urinary total carnitine excretion and plasma total carnitine concentrations were significantly lower in MCAD patients than in controls while they were on normal diets for age. The very low plasma carnitine concentration and urinary excretion rate in the LCAD patient reflect the fact that he was receiving a carnitine-free formula. Acylcarnitines composed $60 \%$ of total carnitine excretion in controls and higher percentages in the patients; in three of the patients, all of the urinary carnitine was esterified. However, despite their low plasma concentrations of free carnitine, free carnitine ac-

Table 1. Plasma carnitine concentrations and urinary carnitine excretion rates in patients with acyl-CoA oxidation disorders while not receiving carnitine treatment (mean $\pm S E M$ )

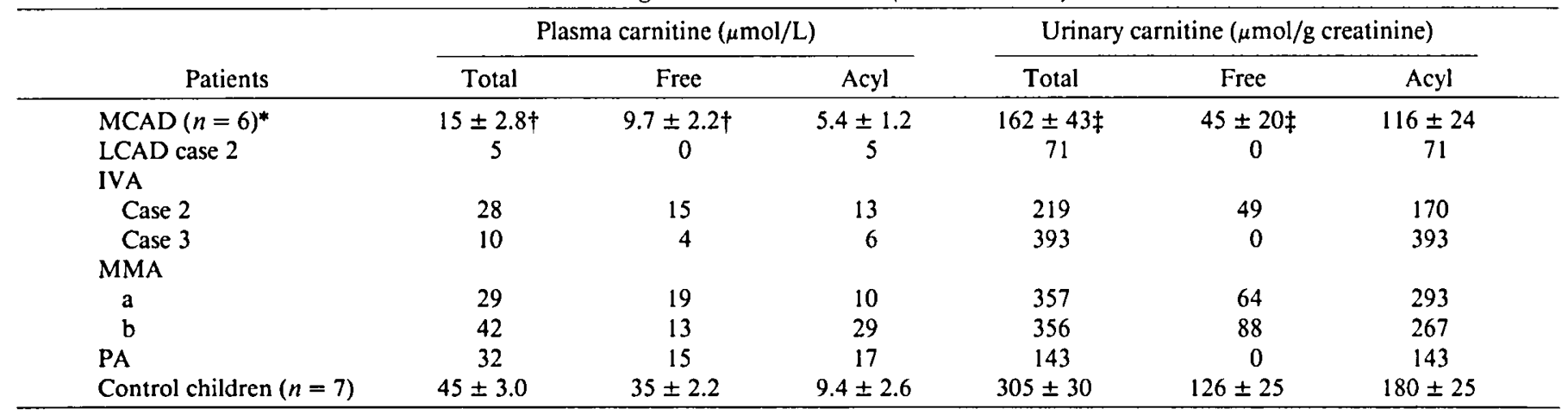

\footnotetext{
* Includes MCAD cases 1 and 2.

$\dagger p<0.01$ vs controls.

$\ddagger p<0.05$ vs controls.
} 
counted for $20-30 \%$ of total urinary carnitine in the MCAD and MMA patients and in one of the IVA patients.

The response to acute withdrawal of oral carnitine was first examined in 10 control adults who were on normal dietary intakes of carnitine. During $5 \mathrm{~d}$ on a carnitine-free diet, there was a small decrease in plasma total $(44 \pm 6.6$ versus $40 \pm 4.3$ $\mu \mathrm{mol} / \mathrm{L}$; mean $\pm \mathrm{SD}, p<0.05)$ and free $(40 \pm 5.8$ versus $34 \pm$ $3.7 \mu \mathrm{mol} / \mathrm{L} ; p<0.01)$ carnitine concentrations and a small, but not statistically significant, decrease in urinary carnitine excretion (total, $178 \pm 54$ versus $143 \pm 43$; free, $86 \pm 38$ versus $58 \pm$ $30 \mu \mathrm{mol} / \mathrm{g}$ creatinine). The mean urinary total carnitine excretion rate in healthy adults was $60 \%$ of that in control children (Table 1). The plasma and urinary acylcarnitine to total carnitine ratio was higher in children than in adults, probably reflecting the influence of $12 \mathrm{~h}$ of fasting in some of the children (18).

The response to acute withdrawal of large doses of exogenous carnitine in the control child is shown in Figure 1 and Table 2. Plasma free and total carnitine concentrations were slightly elevated and urinary total carnitine excretion was 10 times normal during treatment with oral carnitine. Urinary carnitine excretion quickly fell toward the upper end of the normal range by $48 \mathrm{~h}$ and plasma total carnitine declined toward the normal range. Both plasma and urine total carnitine values remained slightly above the means of untreated control children at the end of the study, suggesting that the effect of high-dose carnitine supplements had not been entirely eliminated. This is consistent with observations that 6-8 d are required before reductions in carnitine intake are fully reflected by changes in urinary excretion rates (19). In contrast to this normal response to carnitine withdrawal, there was a rapid redevelopment of severe plasma total carnitine deficiency in the patient with a genetic defect in carni-
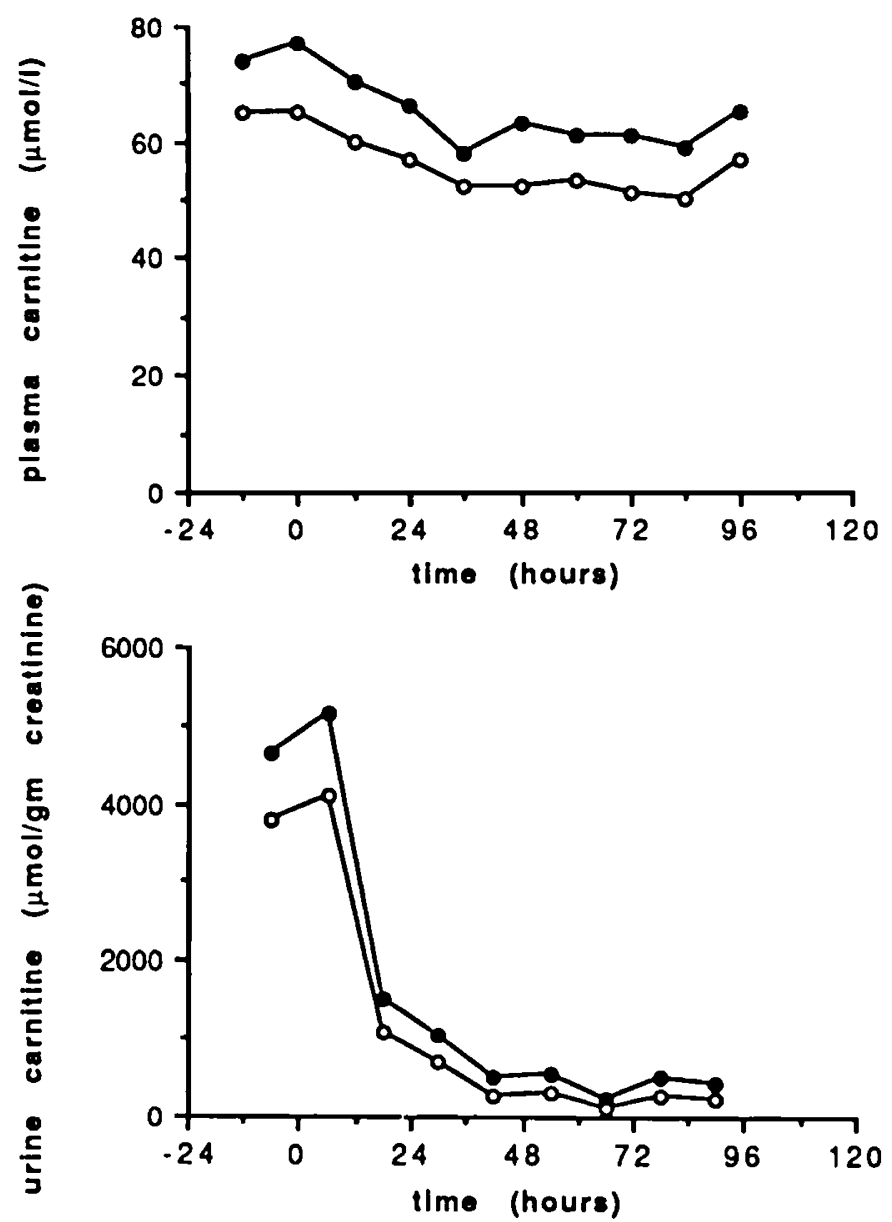

Fig. 1. Response to withdrawal of oral carnitine therapy in a control child. Shown are plasma and urinary free $(O)$ and total $(\bullet)$ carnitine values after discontinuation of carnitine at $0 \mathrm{~h}$. tine transport affecting kidney and muscle (9) in whom renal conservation of carnitine is absent (Fig. 2).

The responses to withdrawal of carnitine supplementation in patients with defects in acyl-CoA oxidation are shown in Table 2. Figures 3 to 6 illustrate the time course in examples of each disorder. During treatment, plasma total carnitine concentrations were increased to or above the normal range and urinary total carnitine excretion was elevated from 5 to 30 times compared with the mean values of untreated control children (or control adults, in the adolescent MCAD case 2). After carnitine was withheld, urine total carnitine excretion rates rapidly fell and, with the exception of the patient with PA, had reached levels similar to those of untreated controls by $4 \mathrm{~d}$. In the PA patient, total carnitine excretion fell from rates 30 times normal to a value 3 times normal. Plasma total carnitine concentrations also fell quickly in the patients, and in the MCAD and IVA patients had plateaued by $\mathrm{d} 4$ at subnormal values comparable to those seen in the untreated patients (Table 1). In the LCAD patients, the fall in plasma total carnitine levels stabilized by $\mathrm{d} 4$ at values in the lower normal range but below that of the treated control child shown in Figure 1. In the PA patient, plasma total carnitine fell from very high levels to reach the upper normal range at $4 \mathrm{~d}$.

Plasma acylcarnitine concentrations were elevated during carnitine treatment in patients with LCAD, IVA, and, particularly, PA, but were not increased in the MCAD patients compared with both untreated controls and the treated control child. With the exception of MCAD case 1, urine from the patients contained a higher proportion of acylcarnitine throughout the study compared with the control child. This was especially dramatic in the PA patient, in whom approximately $80 \%$ of urinary carnitine was esterified at all times. After $4 \mathrm{~d}$, this patient, whose plasma total carnitine values had not yet fallen below normal, continued to excrete acylcarnitines at rates 4 times that of untreated controls. In all of the other patients, $4 \mathrm{~d}$ after stopping carnitine, the rates of acylcarnitine excretion had fallen to values similar to those of untreated controls.

Throughout the withdrawal period, all of the patients continued to excrete free carnitine in the urine, although plasma free carnitine concentrations had fallen to levels well below the normal range in the MCAD, IVA, and PA patients. Because similar inappropriate excretion of free carnitine also occurs with defective carnitine transport (Fig. 2), the relationship between plasma free carnitine and urinary clearance of free carnitine was examined. Figure 7 illustrates this relationship in the control child and patients with IVA, MCAD, and LCAD. During carnitine supplementation, the clearance of free carnitine was markedly elevated in all eight patients and the control child (10 to $40 \%$ of the filtered load versus $2.4 \pm 1.4 \%$ in untreated control children and $1.8 \pm 0.8 \%$ in untreated adults). The clearance was $90-100 \%$ in the patient with the carnitine transport defect. By 4 $\mathrm{d}$, free carnitine clearance had decreased to $1-4 \%$ in all of the patients and the control but remained greater than $100 \%$ in the carnitine transport defect.

Table 3 shows the estimated renal thresholds for free carnitine, defined as the plasma free carnitine level at which clearance fell to $<5 \%$. Because the normal mean fractional excretion of free carnitine is only $1-2 \%$, the method tends to overestimate the threshold. This may partly account for fact that the value of 56 $\mu \mathrm{mol} / \mathrm{L}$ found in the control child was considerably higher than the value of $35-40 \mu \mathrm{mol} / \mathrm{L}$ found in normal subjects by Engel et al. (12) using carnitine loading. Moreover, as noted above, the effects of prior carnitine treatment had not disappeared completely in this child by the end of the study period. Patients with all four different acyl-CoA oxidation defects had renal thresholds for free carnitine that were intermediate between the control child and the patient with the carnitine transport defect (Table 3). The MCAD, IVA, and PA patients had estimated renal thresholds for free carnitine between 10 and $25 \mu \mathrm{mol} / \mathrm{L}$, well below either that of the control child or the range found by the 
Table 2. Plasma carnitine concentrations and urinary carnitine excretion rates before and after withdrawal of carnitine treatment

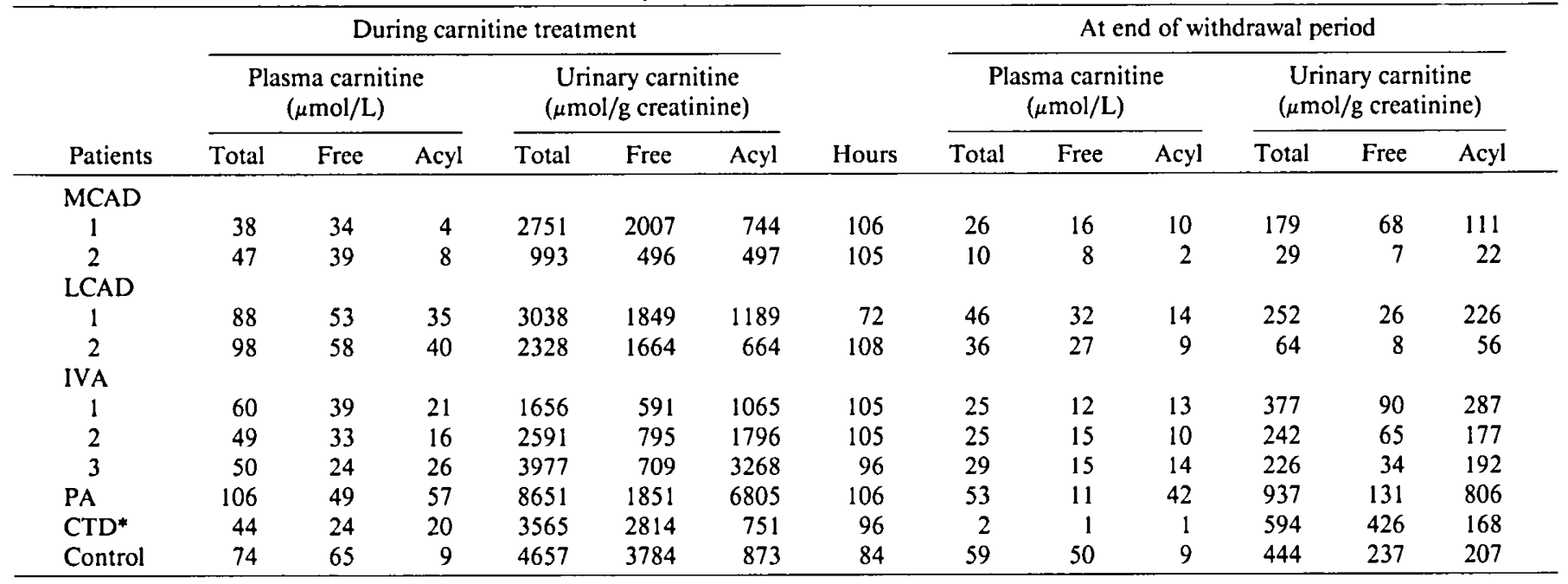

* CTD, carnitine transport defect.
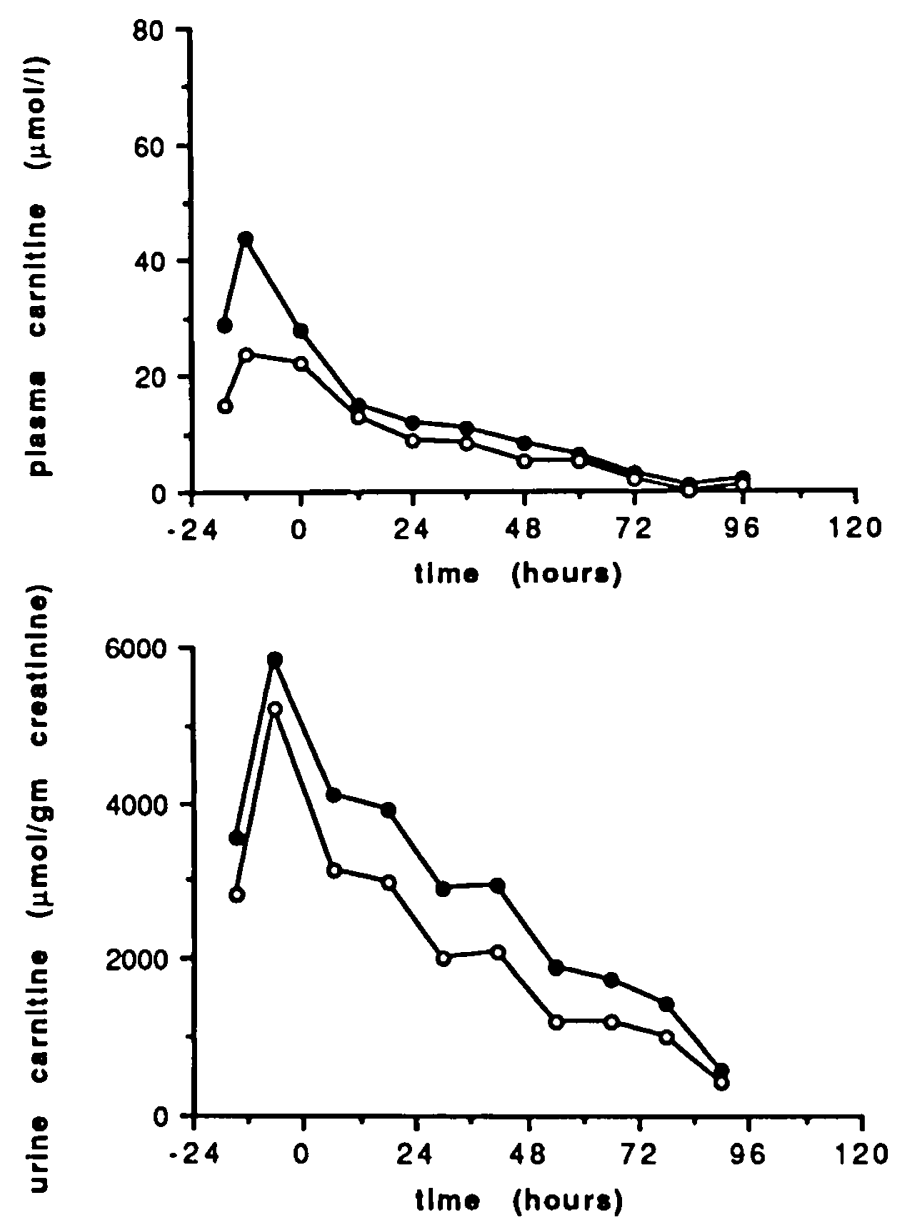

Fig. 2. Response to withdrawal of oral carnitine therapy in a patient with a genetic defect in plasma membrane transport of carnitine. Shown are plasma and urinary free $(O)$ and total $(0)$ carnitine values after discontinuation of carnitine at $0 \mathrm{~h}$. Redrawn from Treem et al. (9) with permission.

carnitine infusion method. In both of the LCAD patients, the estimated renal thresholds for free carnitine were lower than that of the control child but not below those reported by Engel $e t$ al. (12). There was no evidence of other renal tubular dysfunction such as glucosuria or phosphate or bicarbonate wasting in any
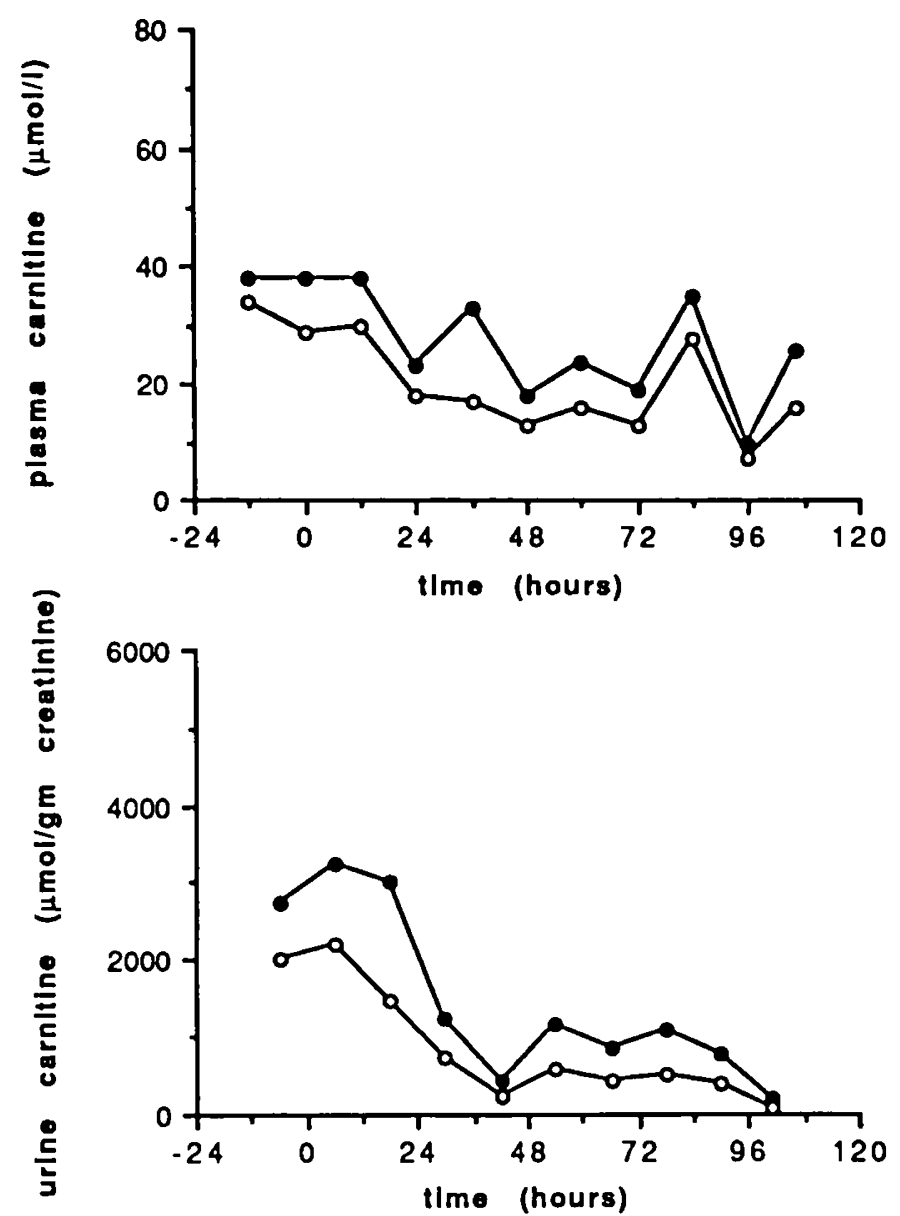

Fig. 3. Response to withdrawal of oral carnitine therapy in MCAD (case 1). Shown are plasma and urinary free $(O)$ and total $(\Theta)$ carnitine values after discontinuation of carnitine at $0 \mathrm{~h}$.

of the patients, and in vitro studies have shown that the carnitine transport system is normal in MCAD and LCAD (9).

As shown in Figure 3, marked fluctuations in plasma free and total carnitine concentrations were found in MCAD case 1 during the latter part of the withdrawal study. Values were lower at $0800 \mathrm{~h}$, after overnight fasting, than at $2000 \mathrm{~h}$. A similar diurnal pattern was observed over $5 \mathrm{~d}$ in a 9-y-old MCAD patient 

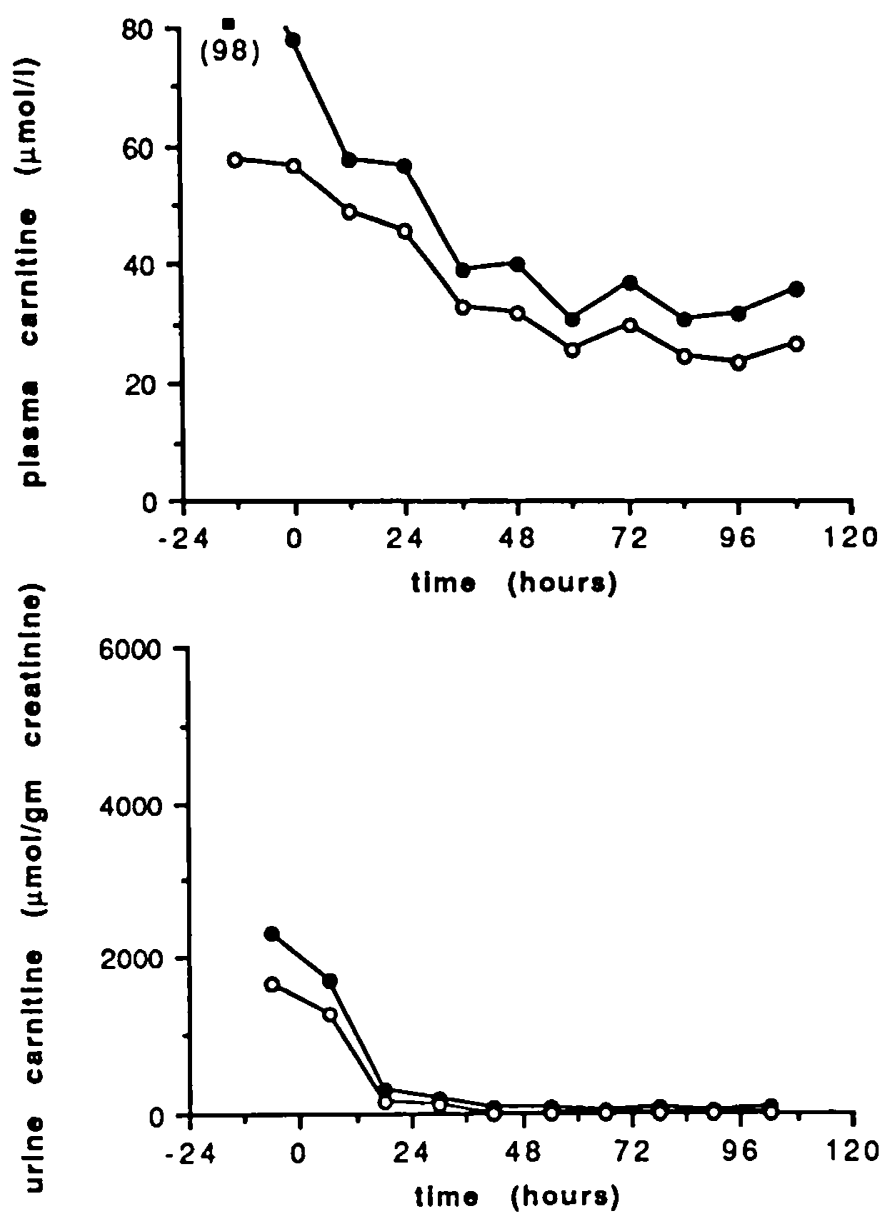

Fig. 4. Response to withdrawal of oral carnitine therapy in LCAD (case 2). Shown are plasma and urinary free $(O)$ and total $(O)$ carnitine values after discontinuation of carnitine at $0 \mathrm{~h}$.

who was not receiving carnitine, but was not apparent in the adolescent MCAD case 2 (data not shown). In neither of the two MCAD patients with variable plasma carnitine concentrations did the times of lower plasma carnitine levels coincide with increases in the rates of urinary acylcarnitine or total carnitine excretion. These observations suggest the possibility of diurnal fluctuations in the renal free carnitine threshold, although this explanation could not be tested from the data available.

\section{DISCUSSION}

The processes responsible for regulating body stores of carnitine in normal individuals are outlined in Figure $8(1)$. Over $95 \%$ of total carnitine in the body is maintained intracellularly through sodium gradient-dependent transport across the plasma membrane. Because carnitine is not degraded, turnover is limited to input from endogenous synthesis plus dietary sources, such as meats and milk, and output through urinary excretion. Approximately $75 \%$ of new carnitine is usually provided from the diet, based on the differences in urinary total carnitine excretion rates between individuals on normal and on carnitine-free diets for prolonged periods $(19,20)$. The remaining $25 \%$ comes from endogenous synthesis of carnitine from trimethyllysine residues released during protein turnover. Total body stores are controlled chiefly by the kidney, which reabsorbs $98-99 \%$ of filtered free carnitine at normal plasma concentrations of carnitine. Except for long-chain acylcarnitines, which are more hydrophobic and may be bound to plasma proteins, the renal clearance of acylcarnitines is greater than that of free carnitine, as witnessed by the higher proportions of acylcarnitines in urine than in plasma.
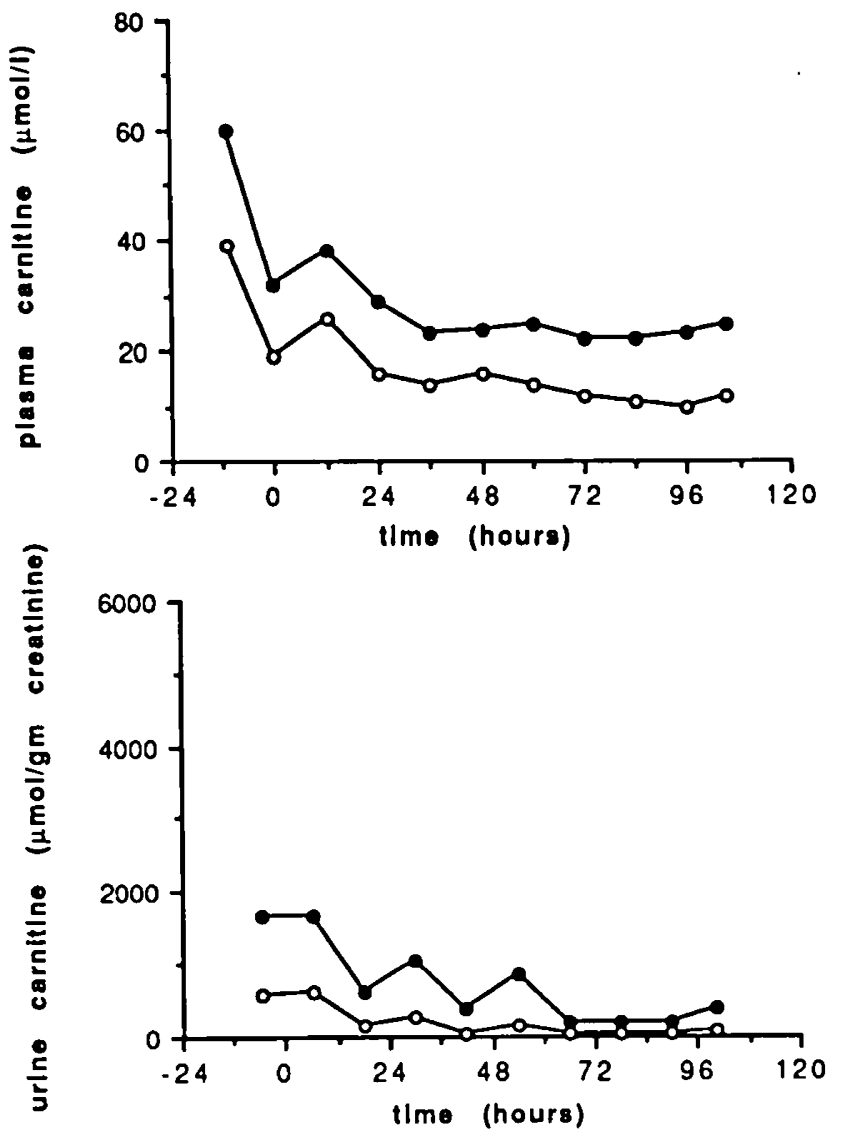

Fig. 5. Response to withdrawal of oral carnitine therapy in IVA (case $2)$. Shown are plasma and urinary free $(O)$ and total $(\bullet)$ carnitine values after discontinuation of carnitine at $0 \mathrm{~h}$.

Panels 1 and 2 in Figure 8 outline the two alternative mechanisms that have been proposed to explain secondary carnitine deficiency in patients with defects in acyl-CoA oxidation. In the acylcarnitine wastage model, exemplified by chronic administration of pivalate $(6,7)$, excess formation of acylcarnitines and their excretion in the urine leads to a chronic depletion of total carnitine stores, because urinary loss constantly exceeds the sum of dietary intake plus synthesis (panel 1). In the impaired carnitine transport model, exemplified by the genetic defect in carnitine transport $(9,10)$, both the renal free carnitine threshold and the tissue gradient are decreased. Normal plasma total carnitine levels can only be maintained by constantly giving large supplements of carnitine (panel 2a); when supplements are stopped, there is a prompt return to the deficient state within a few days without a persistent imbalance between rates of carnitine input and output ( panel $2 b$ ).

The present results of studies in patients with defects in acylCoA oxidation in both the basal state and after acute withdrawal of oral carnitine supplements indicate that the acylcarnitine wastage model is not adequate to entirely explain their secondary carnitine deficiency. Evidence against this model as the sole explanation includes the following: 1 ) total urinary carnitine excretion in the basal state was not elevated compared with that in control children (Table 1);2) after withdrawal of carnitine supplementation, large losses of total carnitine in the urine were not sustained beyond a few days (Table 2); and 3 ) in most cases, in both the basal state and throughout the withdrawal period, there continued to be inappropriate losses of free carnitine in the urine. The rapidity with which both plasma and urinary total carnitine values returned toward baseline after carnitine withdrawal suggested that the ability of the kidney to conserve carnitine was impaired in these patients. Analysis of the with- 


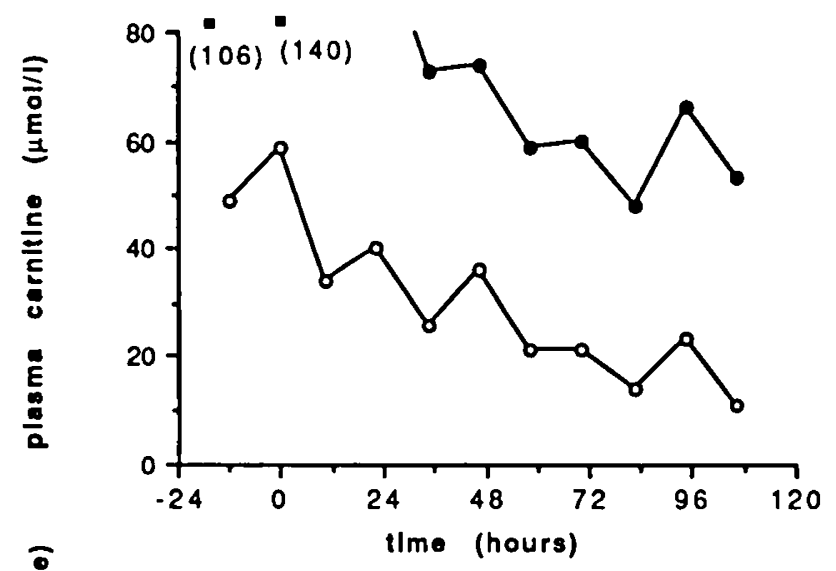

Table 3. Estimated renal thresholds for free carnitine in patients with acyl-CoA oxidation defects

\begin{tabular}{cc}
\hline Defect & $\begin{array}{c}\text { Threshold } \\
(\mu \mathrm{mol} / \mathrm{L})\end{array}$ \\
\hline MCAD & $13-16$ \\
Case 1 & 25 \\
Case 2 & \\
LCAD & 43 \\
Case 1 & 52 \\
Case 2 & \\
IVA & 18 \\
Case 1 & 17 \\
Case 2 & 16 \\
Case 3 & $14-23$ \\
PA & $<2$ \\
Carnitine transport defect & 56 \\
Control child & \\
\hline
\end{tabular}

baseline after carnitine withdrawal in patients with MCAD and IVA than in the control child. Holme et al. $(6,7)$ have reported that continued administration of pivalate results in a sustained high rate of pivaloylcarnitine excretion that progressively depletes plasma and tissue total carnitine concentrations to below $10 \%$ of normal. With usual dietary intakes of carnitine, this severe degree of carnitine deficiency does not occur in patients with acyl-CoA oxidation disorders (Table 1). Their milder degree of carnitine deficiency may be explained partly by equilibration at their reduced renal free carnitine thresholds and partly by the observation that acylcarnitine excretion declines as carnitine levels fall in these patients to reach a point where total carnitine output is in balance with the sum of carnitine intake and synthesis. A corollary of this interpretation is that, unlike patients chronically taking pivalate, the rate of production of nonmetabolizable acyl-CoA in the acyl-CoA oxidation disorders is regulated by the amount of free carnitine available. One effect of carnitine supplementation in these patients may be to increase the rate of acyl-CoA formation as well as the rate of its removal.

Other possible causes of secondary carnitine deficiency in the acyl-CoA oxidation defects, such as increased degradation or decreased input, can be excluded. As noted above, like other $\mathrm{N}$ trimethylated substrates, such as trimethylhistidine, carnitine cannot be degraded in the body, but must be eliminated by excretion in the urine (1). Dietary carnitine deficiency, either acute (see Results) (19) or chronic (20), has little effect on plasma carnitine levels, except in very young infants (21), due to efficient renal conservation of carnitine. Dietary deficiency probably contributed to the very low plasma concentrations of carnitine seen in LCAD case 2 when first studied compared with the values noted at the end of the withdrawal study (Tables 1 and 2). Because rates of endogenous synthesis are only one third of normal dietary intake $(19,20)$ and much smaller than the total body store of carnitine, an impairment of endogenous synthesis could not cause the rapid changes in plasma carnitine levels found in this study. In addition, the patients had no evidence of impairment in other renal tubular functions to suggest that their carnitine deficiency was part of a renal Fanconi syndrome (11).

The rates of urinary total and acylcarnitine excretion found in drawal responses showed decreased renal thresholds for free carnitine, implying an alteration in renal transport of free carnitine.

It should be emphasized that the proportion of acylcarnitines in urine from patients was elevated in the basal state as well as during and after the period of carnitine supplementation. This indicates that excretion of specific abnormal acylcarnitines is likely to play a role in the reduction of total carnitine concentrations in some of these disorders in addition to that of lowered renal free carnitine threshold. For example, the contribution of abnormal acylcarnitines, such as octanoyl and isovalerylcarnitine, to total carnitine excretion may account for the observation that plasma carnitine concentrations returned more quickly to the present study before carnitine supplementation in the different acyl-CoA oxidation disorders are similar to those previously reported in affected children by Chalmers et al. (22). Based on comparison with values in adult controls, these workers suggested that their patients had excessive rates of total carnitine excretion. In contrast, the present results showed that total carnitine excretion in the patients was not abnormal when children were used as controls. As noted in the Results, the mean urinary total carnitine excretion rate relative to creatinine was $70 \%$ higher in control children than in control adults.

An attractive model for the reduced renal thresholds for free carnitine found in the acyl-CoA oxidation defects in the present 
1.

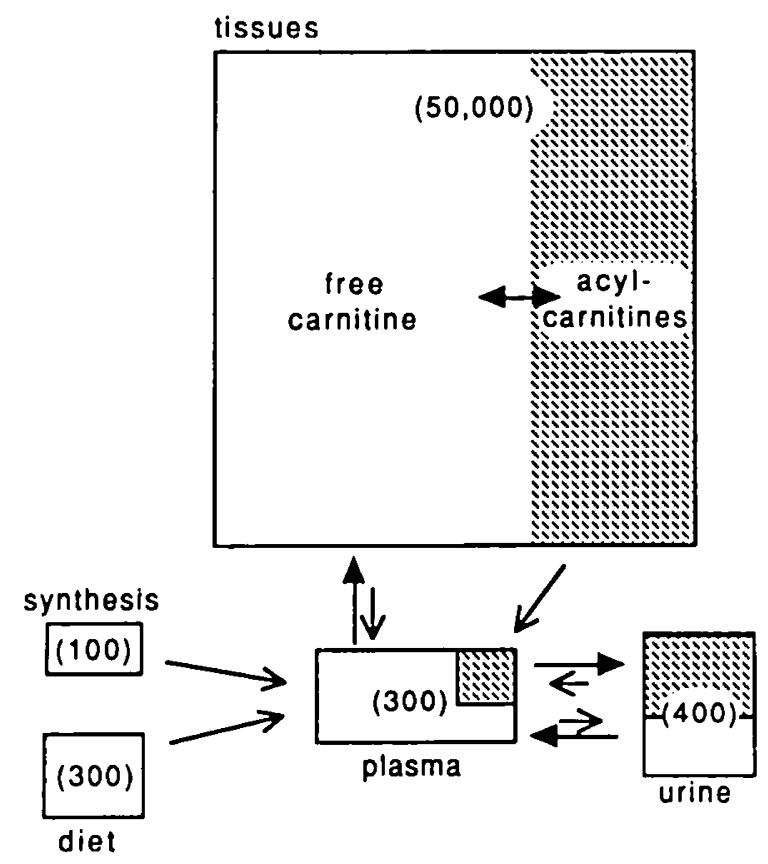

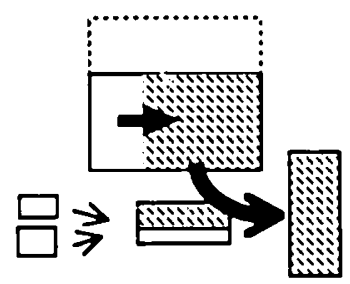

2.

(a)

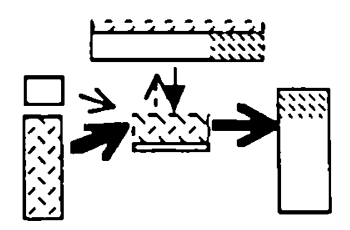

(b)

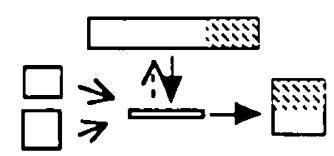

Fig. 8. Regulation of body stores of carnitine in normal subjects (left panel) and in the models of acylcarnitine wastage ( $I$ ) and impaired carnitine transport with $(2 a)$ and without $(2 b)$ carnitine supplementation. Approximate sizes of total carnitine pools and 24 -h input and output rates are given in $\mu \mathrm{mol}$.

study is that they arise from inhibition of free carnitine transport by accumulated acylcarnitines. This hypothesis is supported by studies in cultured fibroblasts showing that acylcarnitines potently inhibit the plasma membrane transport system for free carnitine that is expressed in muscle and kidney (10). Whether inhibition of renal free carnitine transport is due to intracellular or extracellular acylcarnitines and whether free carnitine uptake or secretion is affected remain to be established. Inhibitory effects of acylcarnitines on free carnitine transport in other tissues might also contribute to the low levels of carnitine found in muscle and liver in patients with acyl-CoA oxidation defects (2). The hypothesis is consistent with observations that secondary carnitine deficiency is a feature common to all of the known fatty acid oxidation defects that lead to accumulation of acylcarnitines (23), whereas plasma carnitine concentrations and the renal free carnitine threshold are elevated in carnitine palmitoyltransferase1 deficiency, the one defect in which acylcarnitine formation is blocked (24). A partial, but incomplete, inhibition of free carnitine transport by acylcarnitines is consistent with the observation that patients with acyl-CoA oxidation defects have an intermediate degree of carnitine deficiency that is stable over time (Table 1) similar to that seen in heterozygous parents of patients with the genetic carnitine transport defect (10).

The above hypothesis provides an explanation for the fluctuations in plasma total carnitine concentrations seen in MCAD case 1 and a second, untreated MCAD patient. Variability in plasma total carnitine has been previously commented upon by Rebouche and Engel (13) in four patients who were later found to have MCAD $(2,13)$. In our two patients, plasma levels of free and total carnitine were lower after an overnight fast than after a day of eating. The greatest accumulation of inhibitory mediumchain acylcarnitines would be expected to occur during the overnight fast, leading to lower renal free carnitine thresholds and lower plasma free and total carnitine levels at $0800 \mathrm{~h}$ than at $2000 \mathrm{~h}$. In addition, the hypothesis provides a possible explanation for the observation that MCAD patients may demonstrate an increase in plasma as well as in urinary concentrations of free, acyl, and total carnitine during fasting $(3,25)$. The increase in plasma free carnitine might reflect release from tissues, because tissue carnitine transport is inhibited by the elevated levels of medium- and long-chain acylcarnitines associated with fasting. Increased urinary excretion may reflect the combined effects of higher plasma levels of carnitine, greater inhibition of renal carnitine transport, and higher rates of medium-chain acylcarnitine loss.

The relative contributions to carnitine deficiency of the lowered renal thresholds for free carnitine and the excretion of abnormal acylcarnitines in the different acyl-CoA oxidation defects are difficult to separate, but probably vary depending on the specific disorder as well as the clinical status of the patient. Impaired free carnitine transport may be the sole factor in patients with LCAD and other long-chain fatty acid oxidation disorders, inasmuch as few or no long-chain acylcarnitines appear in their urine $(8,23)$. At the time of the withdrawal study, the two LCAD patients were on intensive feeding regimens that would have suppressed lipolysis and the formation of long-chain acylcarnitines. This might account for the fact that they were able to maintain plasma concentrations of carnitine close to normal, although very low levels have been observed in LCAD patients at initial presentation (26). We have recently found normal plasma concentrations of carnitine in an adult LCAD patient, but have also found markedly impaired renal carnitine conservation in a child with deficiency of carnitine/acylcarnitine translocase (23). Specific abnormal acylcarnitines have been demonstrated in urine from MCAD, IVA, and PA patients. Isovalerylcarnitine and propionylcarnitine have been reported to 
account for most of the urinary esterified carnitine in IVA and PA patients $(4,27)$, but medium-chain acylcarnitines may comprise less than half of the urinary esterified carnitine in MCAD patients (25). This suggests that excretion of abnormal acylcarnitines may be more important in sustaining carnitine deficiency in IVA and particularly PA than in MCAD or LCAD patients. It should be noted, however, that the untreated PA and MMA patients and the treated PA patient had more pronounced accumulations of acylcarnitines in plasma and urine but higher plasma levels of free and total carnitine compared with MCAD patients. This might reflect differences in the effects of propionyl and medium-chain acylcarnitines on the carnitine transport system, inasmuch as we have found that medium- and long-chain acylcarnitines inhibit transport more potently than the shortchain ester, acetylcarnitine (10).

In summary, the present studies indicate that secondary carnitine deficiency in the acyl-CoA oxidation defects is not solely due to acylcarnitine wastage. They suggest that the deficiency arises from indirect, as well as direct effects of accumulated acylcarnitines. In contrast to the progressive severe carnitine deficiency induced by chronic administration of pivalate, patients with these disorders maintain a partial deficiency reflecting both lowered renal thresholds for free carnitine and rates of excretion of abnormal acylcarnitines that are not out of balance with rates of carnitine input. The rapid decline in acylcarnitine excretion after withdrawal of carnitine supplements to rates similar to those in control children suggests that treatment with carnitine causes a parallel increase in both the rate of formation and the rate of removal of nonmetabolizable acyl-CoA in these defects.

Currently, the rationale for carnitine therapy in the acyl-CoA oxidation disorders is based almost entirely on theoretical considerations. One hypothesis is that tissue concentrations of free carnitine become so low as to limit one or more carnitinedependent pathways (e.g. long-chain fatty acid oxidation). There is little evidence for this postulate. Reported tissue values in these disorders are considerably higher than in the carnitine transport defect or with pivalate administration $(2,7,10)$. One report indicates that the degree of free carnitine deficiency associated with PA and MMA is not severe enough to impair hepatic fatty acid oxidation (28). In addition, the present study suggests that the free carnitine deficiency in a wide range of acyl-CoA oxidation defects is stable and partial, rather than progressive and severe. The second hypothesis is that carnitine treatment, via the reversible acylcarnitine transferase reaction, would lower intramitochondrial levels of acyl-CoA intermediates that might accumulate behind the specific metabolic blocks, thus relieving the potential toxic effects of these compounds. This hypothesis has been suggested to explain the improvement in fasting tolerance reported with carnitine treatment in patients with PA and MMA (28). Nevertheless, evidence for the second hypothesis is chiefly indirect and limited to changes in ratios of plasma or urinary total acylcarnitine to free carnitine concentrations (e.g. Table 2); direct measurements have not been made of tissue concentrations of specific acyl-CoA intermediates. The second hypothesis is not proven by increases in abnormal acylcarnitine excretion with carnitine therapy inasmuch as, as noted above, this could reflect increased production of the corresponding abnormal acyl$\mathrm{CoA}$ without a clinically significant change in its steady state tissue concentrations. Finally, although it is generally assumed that treatment with carnitine is benign even if not effective, concern has been raised that its use might worsen toxicity from long-chain acylcarnitines in some fatty acid oxidation defects $(23,29)$. Whether carnitine treatment is beneficial in one or more of the acyl-CoA oxidation disorders, either in altering tissue concentrations of toxic intermediates or in other ways, requires additional controlled studies.

Acknowledgments. The authors thank Dr. Dennis J. McGarry for first suggesting an examination of the development of sec- ondary carnitine deficiency, Niel Turner and Susan Deleeuw for technical assistance, Norma Govens for helping with the manuscript, and the nurses in the Clinical Research Center for expert care of the children in this study.

\section{REFERENCES}

1. Bremer J 1983 Carnitine: metabolism and functions. Physiol Rev 63:14201480

2. Stanley CA 1987 New genetic defects in mitochondrial fatty acid oxidation and carnitine deficiency. Adv Pediatr 34:59-88

3. Stanley CA, Hale DE, Coates PM, Hall CL, Corkey BE, Yang W, Kelley RI, Gonzales ES, Williamson JR, Baker L 1983 Medium-chain acyl-CoA dehydrogenase deficiency in children with non-ketotic hypoglycemia and low carnitine levels. Pediatr Res 17:877-884

4. Roe CR, Millington DS, Maltby DA, Kahler SG, Bohan TP 1984 L-Carnitine therapy in isovaleric acidemia. J Clin Invest 74:2290-2295

5. Roe CR, Coates PM 1989 Acyl-CoA dehydrogenase deficiencies. In: Scriver CR, Beaudet AL, Sly WS, Valle D (eds) The Metabolic Basis of Inherited Disease. McGraw-Hill, New York, pp 889-914

6. Holme E, Jacobson CE, Nordin I, Greter J, Lindstedt S, Kristiansson B, Jodal U 1989 Carnitine deficiency induced by pivampicillin and pivmecillinam therapy. Lancet 2:469-473

7. Holme E, Jodal U, Linstedt S, Nordin I 1992 Effects of pivalic acid-containing prodrugs on carnitine homeostasis and on response to fasting in children. Scand J Clin Lab Invest 52:361-372

8. Roe CR, Millington DAM, Bohan TP, Kahler SG, Chalmers RA 1985 Diagnostic and therapeutic implications of medium-chain acylcarnitines in the medium-chain acyl-CoA dehydrogenase deficiency. Pediatr Res 19:459-466

9. Treem WR, Stanley CA, Finegold DN, Hale DE, Coates PM 1988 Primary carnitine deficiency due to a failure of carnitine transport in kidney, muscle, and fibroblasts. N Engl J Med 319:1331-1336

10. Stanley CA, DeLeeuw S, Coates PM, Vianey-Liaud C, Divry P, Bonnefont JP, Saudubray JM, Haymond M, Trefz FK, Brenningstall GN, Wappner RS, Byrd DJ, Sansaricq C, Tein I, Grover W, Valle D, Rutledge SL, Treem WR 1991 Chronic cardiomyopathy and weakness or acute coma in children with a defect in carnitine uptake. Ann Neurol 30:709-716

11. Bernardini I, Rizzo WM, Dalakas M, Bernar J, Gahl WA 1985 Plasma and muscle free carnitine deficiency due to renal Fanconi syndrome. J Clin Invest 75:1124-1130

12. Engel AG, Rebouche CJ, Wilson DM, Glasgow AM, Romshe CA, Cruse RP 1981 Primary systemic carnitine deficiency: II. Renal handling of carnitine. Neurology 31:819-825

13. Rebouche CJ, Engel AG 1984 Kinetic compartmental analysis of carnitine metabolism in the human carnitine deficiency syndromes. Evidence for alterations in tissue carnitine transport. J Clin Invest 73:857-867

14. Matsubara Y, Narisawa K, Miyabayashi S, Tada K, Coates PM 1990 Molecular lesion in patients with medium-chain acyl-CoA dehydrogenase deficiency. Lancet 335:1589

15. Yokota I, Indo Y, Coates PM, Tanaka K 1990 Molecular basis of mediumchain acyl-coenzyme $A$ dehydrogenase deficiency. An $A$ to $G$ transition at position 985 that causes a lysine-304 to glutamate substitution in the mature protein is the single prevalent mutation. J Clin Invest 86:1000-1003

16. Kelly DP, Whelan AJ, Ogden ML, Alpers R, Zhang ZF, Bellus G, Gregersen N, Dorland L, Strauss AW 1990 Molecular characterization of inherited medium-chain acyl-CoA dehydrogenase deficiency. Proc Natl Acad Sci USA 87:9236-9240

17. McGarry JD, Foster DW 1976 An improved and simplified radioisotopic assay for the determination of free and esterified carnitine. J Lipid Res 17:277281

18. Hoppel CL, Genuth SM 1982 Urinary excretion of acetylcarnitine during human diabetic and fasting ketosis. Am J Physiol 243:E168-E172

19. Rudman D, Sewell CW, Ansley JD 1977 Deficiency of carnitine in cachectic cirrhotic patients. J Clin Invest 60:716-723

20. Lombard KA, Olson AL, Nelson SE, Rebouche CJ 1989 Carnitine status of lactoovovegetarians and strict vegetarian adults and children. Am J Clin Nutr 50:301-306

21. Schmidt-Sommerfeld E, Penn D 1990 Carnitine and total parenteral nutrition in the neonate. Biol Neonate 58(suppl 1):81-88

22. Chalmers RA, Roe CR, Stacey TE, Hoppel CL 1984 Urinary excretion of $L$ carnitine and acylcarnitines by patients with disorders of organic acid metabolism. Pediatr Res 18:1325-1328

23. Stanley CA, Hale DE, Berry GT, Deleeuw S, Boxer J, Bonnefont JP 1992 A deficiency of carnitine-acylcarnitine translocase in the inner mitochondrial membrane. N Engl J Med 327:19-23

24. Stanley CA, Sunaryo F, Hale DE, Bonnefont JP, DeMaugre F, Saudubray JM 1992 Elevated plasma carnitine in the hepatic form of carnitine palmitoyltransferase-1 deficiency. J Inherited Metab Dis 15:785-789

25. Schmidt-Sommerfeld E, Penn D, Kerner J, Bieber LL, Rossi TM, Lebenthal E 1989 Quantitation of urinary carnitine esters in a patient with mediumchain acyl-coenzyme A dehydrogenase deficiency: effect of metabolic state and L-carnitine therapy. J Pediatr 115:577-582 
26. Hale DE, Batshaw ML, Coates PM, Frerman FE, Goodman SI, Singh I, Stanley CA 1985 Long-chain acyl coenzyme A dehydrogenase deficiency: an inherited cause of nonketotic hypoglycemia. Pediatr Res 19:666-671

27. Davies SE, Iles RA, Stacey TE, Sousa CD, Chalmers RA 1991 Carnitine therapy and metabolism in the disorders of propionyl-CoA metabolism studied using 1H-NMR spectroscopy. Clin Chim Acta 204:263-277
28. Wolff JA, Carroll JE, Le PT, Prodanos C, Haas R, Nyhan WL 1986 Carnitine reduces fasting ketogenesis in patients with disorders of propionate metabolism. Lancet 1:289-291

29. Corr PB, Creer MH, Yamada KA, Saffitz JE, Sobel BE 1989 Prophylaxis of early ventricular fibrillation by inhibition of acylcarnitine accumulation. $\mathrm{J}$ Clin Invest 83:927-936

\section{Announcement}

\section{The Australian NHMRC Twin Registry: A Resource for Pediatric Research}

The Australian NHMRC Twin Registry contains more than 4000 pairs of twins under 10 years old and 3000 more up to the age of 18 years. The parents of these twins have volunteered to consider requests from researchers for studies in bona fide projects approved by the Registry. This represents a major resource for studies in pediatric and adolescent epidemiology. There are numerous ways in which twins can be used to address scientific and medical questions. Some examples include examining genetic and environmental variation, co-twin control studies based on disease discordant or exposure discordant pairs, longitudinal studies, and studies of geneenvironment interaction (Clifford and Hopper: The Australian NHMRC Twin Registry. A resource for the Australian scientific community. Med J Aust 60:149, 1986). Although there have been more than 70 projects conducted over the last 12 years on the 17000 adult pairs in the Registry, to date there have been few studies making use of the younger pairs. Baseline information has been computerized on about $50 \%$ of these pairs. Applications to use the Registry can be made from researchers throughout the world. There may be some charge, depending on costs and level of work required by local staff. All applications are reviewed by the Executive Committee of the Registry, and advice on study design and practicalities is available. For more information. contact: Dr. John L. Hopper, Director, Australian NHMRC Twin Registry, 151 Barry Street, Carlton, Victoria 3053, Australia. 